Planetary Systems in the Universe - Observation, Formation and Evolution

Proceedings IAU Symposium No. 202, (c)2004 IAU

Alan Penny, Pawel Artymowicz, Anne-Marie Lagrange, \& Sara Russell, eds.

\title{
The McDonald Observatory Planetary Search Program: Past, Present, and Future
}

\author{
William D. Cochran \\ Department of Astronomy, The University of Texas at Austin, Austin \\ $T X$ 78712, USA \\ Artie P. Hatzes \\ Thüringer Landessternwarte Tautenburg, Sternwarte 5, D-07778 \\ Tautenburg, Germany
}

\begin{abstract}
We present a summary of the McDonald Observatory Planetary Search program. We first summarize results on the star $\epsilon$ Eridani, in which we combine McDonald Observatory data with data from CFHT, Lick, and ESO, to detect a planet in orbit around this very nearby star. We then concentrate on results from the first 4 years of the Texas Hyades survey using the HIRES spectrograph on Keck 1. The Hyades constitute a very homogeneous sample of stars, in which the major independent variable is the stellar mass. In this survey, we have achieved $3 \mathrm{~m} \mathrm{~s}^{-1}$ internal error on a sample of about 100 Hyades dwarfs. We discuss the rms radial velocity jitter of the sample stars, and its relationship to spectral type, stellar rotation, and chromospheric activity.
\end{abstract}

\section{Introduction}

The McDonald Observatory Planetary Search (MOPS) program comprises a large, multifacet investigation to detect, characterize, and understand planetary companions to other stars in our galaxy. The MOPS began in 1988 as a high-precision radial velocity survey of bright nearby stars using the McDonald Observatory $2.7 \mathrm{~m}$ coudé spectrograph, but has expanded substantially in size and scope since then. Figure 1 gives a schematic overview of the various aspects of the MOPS.

\subsection{Radial Velocity Programs}

Phase I of the radial velocity program used the telluric $\mathrm{O}_{2}$ lines near $6300 \AA$ as the velocity metric, a technique suggested by Griffin and Griffin (1973). A single order of the $2.7 \mathrm{~m}$ coudé echelle spectrograph (cs12) was isolated onto a TI $800 \times 800 \mathrm{CCD}$ at $R=210,000$. This system gave $20 \mathrm{~m} \mathrm{~s}^{-1}$ precision on stars down to about $V=6$, but suffered from systematic velocity errors, most likely due to prevailing atmospheric winds. We therefore switched to a temperature stabilized $\mathrm{I}_{2}$ cell (Koch \& Wöhl 1984; Libbrecht 1988) as the velocity metric in 1992. This eliminated the systematic errors, and gave a routine radial velocity precision of $15 \mathrm{~m} \mathrm{~s}^{-1}$. This precision was limited by the $9.6 \AA$ bandpass of the spectrum, and by the poor charge-transfer and readout properties of the CCD. To solve these problems, and to achieve substantially improved precision, we 
McDonald Observatory Planet Search

\begin{tabular}{|c|c|c|}
\hline Radial Velocities & $\begin{array}{c}\text { High } \\
\text { Resolution } \\
\text { Spectroscopy }\end{array}$ & $\begin{array}{c}\text { Transit } \\
\text { Photometry }\end{array}$ \\
\hline $\begin{array}{c}\text { McDonald 2.7m Phase I } \\
\text { Coudé spectrograph } 1 \text { - } 02\end{array}$ & \multirow{3}{*}{$\begin{array}{l}\text { Line Profile } \\
\text { Varlations } \\
\text { non-radial } \\
\text { pulsations } \\
\text { (51 Peg, } \tau \text { Boo) }\end{array}$} & \multirow{2}{*}{$\begin{array}{l}\text { McDonald } \\
0.8 \mathrm{~m} \text { PFC }\end{array}$} \\
\hline $\begin{array}{c}\text { McDonald 2.7m Phase II } \\
\text { Coudé spectrograph } 1 \text { - } 12 \\
\end{array}$ & & \\
\hline $\begin{array}{l}\text { McDonald 2.7m Phase III } \\
\text { Coudé spectrograph } 2 \text { - } 12\end{array}$ & & \multirow{3}{*}{$\begin{array}{l}\text { HST - } 47 \text { Tuc } \\
\text { (Gllliland - PI) }\end{array}$} \\
\hline Keck Hyades Survey & \multirow{4}{*}{$\begin{array}{l}\text { Link Between } \\
\text { Radial Velocities } \\
\text { and } \\
\text { Chromospherlc } \\
\text { Activlty } \\
\text { (with S. Saar) }\end{array}$} & \\
\hline Hobby•Eberly Telescope & & \\
\hline ESO CES (Kürster - PI) & & \multirow{2}{*}{$\begin{array}{c}\text { Kepler } \\
\text { (Boruckl - PI) }\end{array}$} \\
\hline ESO VLT (Kürster - PI) & & \\
\hline
\end{tabular}

Figure 1. The various aspects of the McDonald Observatory Planetary Search program.

began Phase III of the radial velocity program in July 1998 , using the $\mathrm{I}_{2}$ cell with the newly installed 2 dcoudé cross-dispersed echelle spectrograph (Tull et al. 1994). Using the full $1200 \AA$ bandpass of the $I_{2}$ absorption band at $R=60,000$ allows routine precision of $3-5 \mathrm{~m} \mathrm{~s}^{-1}$ to be achieved.

In November 1992 we began a collaboration with M. Kürster, K. Dennerl and S. Döbereiner to conduct a southern hemisphere radial velocity survey using the ESO coudé echelle spectrograph, fed by the coudé auxiliary telescope until that facility was closed, and thereafter by the ESO $3.6 \mathrm{~m}$ telescope (Kürster et al. 1994; Hatzes et al. 1996; Kürster et al. 2000). A new survey of southern hemisphere $M$ dwarfs has been started using UVES on the VLT. In 1996, NASA partnership in the Keck Observatory allowed us to begin a new program of radial velocity planet detection using the Keck 1 HIRES with its $\mathrm{I}_{2}$ cell. This program is described below in Section 2.. We also will soon be starting several new radial velocity search programs using the new Hobby•Eberly Telescope and its High Resolution Spectrograph (Sneden et al. 1998).

\subsection{The Planet Around $\epsilon$ Eridani}

We have combined the data from the McDonald Observatory Planetary Search Phases I, II and III, with data from the ESO survey described above, the CFHT survey of Walker et al. (1995), and the Lick Planet Search (Cumming, Marcy, $\&$ Butler 1999) for the nearby K dwarf $\epsilon$ Eridani. These data, which cover the interval 1980.8-2000.0, show convincing variations with a period of $\sim 7$ yrs. A least squares orbital solution using robust estimation gives orbital parameters of period, $P=6.9 \mathrm{yrs}, K=19 \mathrm{~m} \mathrm{~s}^{-1}$, eccentricity $e=0.6$, projected companion mass $M \sin i=0.86 \mathrm{M}_{\text {Jupiter }}$, and semi-major axis $a_{2}=3.3 \mathrm{AU}$. Ca II H \& K Sindex measurements spanning the same time interval show significant variations 
with periods of 3 and $20 \mathrm{yrs}$, yet none at the RV period. If magnetic activity were responsible for the RV variations then it produces a significantly different period than is seen in the Ca II data. Given the lack of Ca II variation with the same period as that found in the RV measurements, the long-lived and coherent nature of these variations, and the high eccentricity of the implied orbit, Keplerian motion due to a planetary companion seems to be the most likely explanation for the observed RV variations. Details of these results for $\epsilon$ Eridani are given by Hatzes et al. (2000).

\subsection{High Spectral Resolution Line Profile Studies}

The McDonald Observatory $2.7 \mathrm{~m}$ 2dcoudé spectrograph can also be operated in a very high resolution mode of $R \sim 200,000$, with limited spectral coverage. We have exploited this unique capability to study spectral line bisectors of the central stars of suspected extrasolar planetary systems. Using this system, we refuted the claims of Gray (1997) and conclusively proved that the observed radial velocity variations in $51 \mathrm{Peg}$ were due to orbital motion and not due to pulsations (Hatzes, Cochran, \& Bakker 1998a; 1998b) We continue to use this facility to investigate the relationship between stellar RV variations and intrinsic stellar variability and chromospheric activity.

\subsection{Planetary Transit Photometry}

In 1999 we began a program of searching for transits of Jupiter-size objects in short period orbits around stars. This program uses the McDonald Observatory $0.8 \mathrm{~m}$ telescope and its Prime Focus Corrector, which images a $46 / \times 46 /$ region of sky onto a $2048 \times 2048 \mathrm{CCD}$. We have also participated in the HST photometric transit study of 47 Tuc (Gilliland et al. 2000) and in the Kepler mission concept (Koch et al. 1998).

\section{The Keck Hyades Survey}

The large aperture of the Keck telescopes provided a unique opportunity to begin a new, highly focused investigation into the physics of planetary system formation. We wanted to examine the dependence of planet formation on the mass of the central star. To ensure that the dominant independent variable is indeed the mass of the star, this investigation requires a very carefully selected homogeneous sample of stars. The Hyades star cluster very nicely provides such a sample. The Hyades are one of the nearest star clusters, providing a good selection of stars over a large range in mass for which high precision velocities can be obtained with Keck. The stars are all the same age $(\sim 650 \mathrm{Myr})$ with the same metallicity $([\mathrm{Fe} / \mathrm{H}]=0.18)$. We have a sample of about 100 Hyades stars ranging from about F8 to M3. We tried very hard to have a fairly uniform distribution of stars as a function of spectral type, and in particular to have an adequate sample of the low-mass stars. Thus most of the observing time is spent on the late $\mathrm{K}$ and the $\mathrm{M}$ stars. Figure 2 gives a color-magnitude diagram for our sample of Hyades dwarfs.

Figure 3 shows velocities obtained for several of our Hyades program stars. The top row shows three stars with constant radial velocities to the precision of 


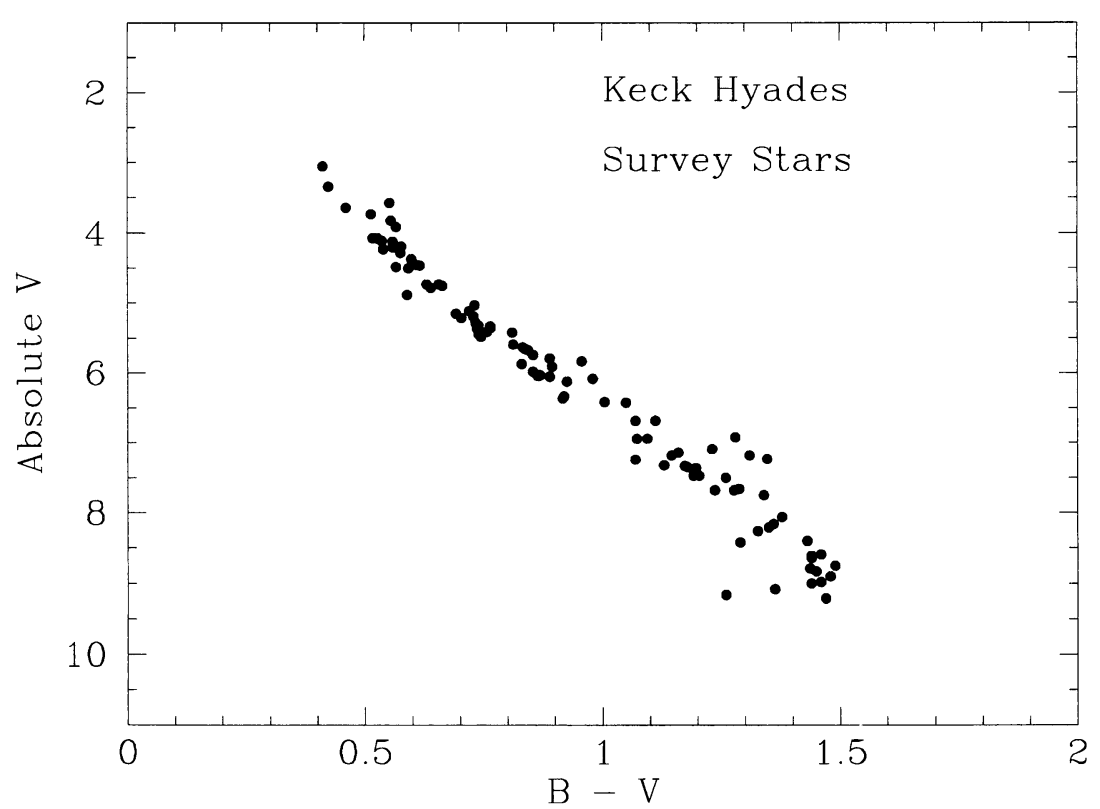

Figure 2. Color magnitude diagram of stars included in the Keck Hyades radial velocity survey. Special effort has been made to include a significant number of low-mass stars in the survey.

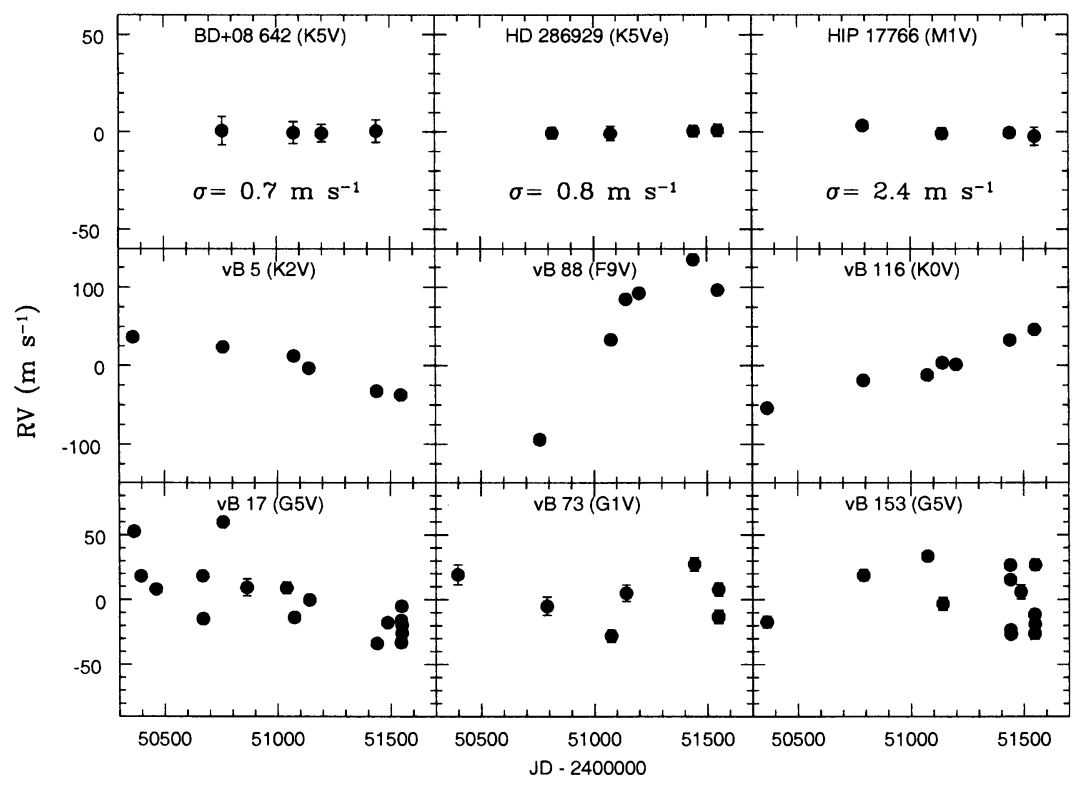

Figure 3. Velocities of several sample stars from the Keck Hyades radial velocity program. The top row shows three stars constant to our measurement precision. The middle row shows three stars with long-term velocity trends, and the bottom row shows three G dwarfs with large RV jitter. 

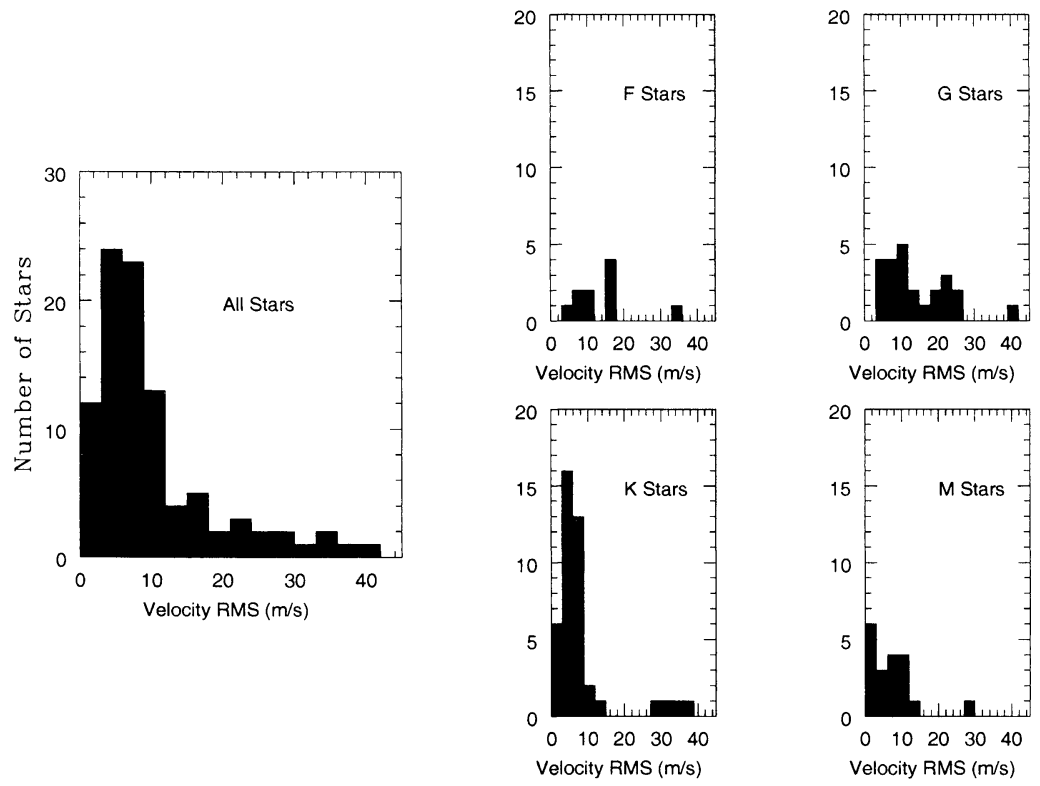

Figure 4. Histogram of the measured velocity rms for all of the program stars (left panel), and for each spectral class separately.

our measurements. These are two K5V stars and an M1V. The second row shows three stars displaying long-term radial velocity trends. None of these three stars have yet completed even half of a complete cycle. If there variations are indeed due to orbital motion, then the companions are probably in the brown dwarf or stellar mass range. The lower row shows three $\mathrm{G}$ dwarfs showing large RV variability. No obvious periodicities are present in these data, but short periods (2-30 days) are poorly sampled. At this time it is unknown whether the observed variability is intrinsic or extrinsic to these stars.

We have now obtained data over four observing seasons for most of the stars in the sample. Figure 4 gives a summary of the rms velocity dispersion for our Hyades sample. The entire sample shows a median rms of about $6 \mathrm{~m} \mathrm{~s}^{-1}$. However, when we break out the sample by spectral type we see some very interesting trends. The $F$ and $G$ stars all show significant velocity variability. None of these stars show an rms below $3 \mathrm{~m} \mathrm{~s}^{-1}$, and only one third of the $\mathrm{F}$ and $\mathrm{G}$ stars show an rms below $9 \mathrm{~m} \mathrm{~s}^{-1}$. No clear periodicities have yet emerged for the $\mathrm{F}$ and $\mathrm{G}$ stars, but the very poor temporal sampling of short periods provided by the pathologies of telescope sampling would make short-period planets very difficult to detect. Follow-up observations of those stars showing significant radial velocity "jitter" are planned for the Hobby•Eberly Telescope High Resolution Spectrograph. The queue scheduled nature of the HET will permit optimal sampling of these very short periods.

The $\mathrm{K}$ and $\mathrm{M}$ stars show a dramatically different picture. The median rms of the velocity measurements decreases significantly among the later spectral types, despite the much fainter apparent magnitudes and the resulting lower SNR. Indeed, about half of the $M$ stars show a velocity rms below $6 \mathrm{~m} \mathrm{~s}^{-1}$. 
The youth of the Hyades dwarfs is manifested in significant chromospheric activity. We set up the Keck HIRES spectrograph so that it automatically includes the spectral region of the Ca II H \& K lines, as well as the 5000-6200 region of the $\mathrm{I}_{2}$ absorption band. Thus, we automatically obtain a simultaneous measurement of the stellar chromospheric activity index for every radial velocity observation. The high velocity $\mathrm{rms}$ of the earlier spectral types appears to be correlated with the stellar rotational $v \sin i$, and is related to the stellar chromospheric activity. These results are in general agreement with the trends seen in nearby field stars by Saar et al. (1998). We are now investigating these effects in much more detail to see if the empirical relationships derived by Saar et al. also apply directly to our Hyades sample. Admittedly, there are some selection effects in our Hyades sample which may affect these results. We have rejected stars with projected rotation velocities $v \sin i$ greater than about $20 \mathrm{~km} \mathrm{~s}^{-1}$. This would prejudice us to slowly rotating stars which might be intrinsically less active, or to rapidly rotating stars viewed pole-on to their rotational axis. We have carefully kept track of all stars considered for our Hyades survey, and we have recorded the reasons for rejecting particular stars. Thus, in the end, we will have a reasonable chance of correcting our results for the known selection effects.

Acknowledgments. This work has been supported by the National Science Foundation LExEn program through grant AST-9808980 and by the NASA Origins Program through grant NAG5-9227.

\section{References}

Cumming, A., Marcy, G. W., \& Butler, R. P. 1999, ApJ, 526, 890

Gilliland, R. L., Brown, T. M., Guhathakurta, P., Sarajedini, A., Milone, E. F., Albrow, M. D., Baliber, N., Bruntt, H., Burrows, A., Charbonneau, D., Choi, P., Cochran, W. D., Edmonds, P. D., Frandsen, S., Howell, J. H., Lin, D. N. C., Marcy, G .W., Mayor, M., Naef, D., Sigurdsson, S., Stagg, C. R., VandenBerg, D. A., Vogt, S. S., \& Williams, M. D. 2000, ApJ, in press

Griffin R., \& Griffin R. 1973, MNRAS, 162, 243

Hatzes, A. P., Cochran, W. D., \& Bakker, E. J. 1998a, Nature, 391, 154

Hatzes, A. P., Cochran, W. D., \& Bakker, E. J. 1998b, ApJ, 508, 380

Hatzes, A. P., Cochran, W. D., McArthur, B., Baliunas, S. L., Walker, G. A. H., Campbell, B., Irwin, A. W., Yang, S., Kürster, M., Endl, M., Els, S., Butler, R. P., \& Marcy, G. W. 2000, ApJ, in press

Koch A. \& Wöhl H. 1984, A\&A, 134, 134

Koch, D. G., Borucki, W., Webster, L., Dunham, E., Jenkins, J., Marriott, J., \& Reitsema, H. J. 1998, Proc. SPIE, 3356, 599

Kürster, M., Hatzes, A. P., Cochran, W. D., Pulliam, C. E., Dennerl, K., \& Döbereiner, S. 1994, ESO Messenger, 76, 51

Kürster, M., Endl, M., Els, S., Hatzes, A. P., Cochran, W. D., Döbereiner, S., \& Dennerl, K. 2000, A\&A, 353, L33

Libbrecht, K. G. 1988, in IAU Symposium 132, The Impact of Very High S/N Spectroscopy on Stellar Physics, ed. G. C. de Strobel \& M. Spite (Dordrecht: Kluwer), 83

Saar, S. H., Butler, R. P., \& Marcy, G. W. 1998, ApJ, 498, L157 
Sneden, C., Tull, R. G., Cochran, W. D., Churchill, C. W., \& Charlton, J. C. 1998, BAAS, 30, 1285

Tull, R. G., MacQueen, P., Sneden, C., \& Lambert, D. L. 1994, in ASP Conf. Ser. Vol. 55, Optical Astronomy from the Earth and Moon, ed. D. M. Pyper \& R. J. Angione (San Francisco: ASP), 148

Walker, G. A. H., Walker, A. R., Irwin, A. W., Larson, A. M., Yang, S. L. S., \& Richardson, D. C. 1995, Icarus, 116, 359 